\title{
Asymmetries in Heavy Meson Production from Light Quark Fragmentation
}

\author{
J. Dias de Deus * and F. O. Durães ${ }^{\dagger \dagger}$ \\ Departamento de Física/CENTRA, IST, \\ 1096 Lisboa Codex, Portugal
}

November 19, 2018

\begin{abstract}
We discuss the possibility of the asymmetry in $D^{-} / D^{+}$production, from $\pi^{-}$beams, being a direct consequence of the properties of the light quark fragmentation function into heavy mesons. The main features of the asymmetry, as a function of $x_{F}$, are easily described. An integrated sum-rule for the $D^{-}, D^{+}$difference, is presented. Predictions for the asymmetry, in $B$ meson production, are given.
\end{abstract}

In the framework of perturbative QCD it is not easy to explain the observed asymmetry in the production of leading and non leading charmed mesons in fixed target experiments with $\pi^{-}$beams [1]. In fact, in the $x_{F} \geq 0$ region, an excess of $D^{-}(d \bar{c})$ over $D^{+}(\bar{d} c)$ is observed, the asymmetry, defined as

$$
A\left(x_{F}\right) \equiv \frac{N^{-}\left(x_{F}\right)-N^{+}\left(x_{F}\right)}{N^{-}\left(x_{F}\right)+N^{+}\left(x_{F}\right)},
$$

increasing with $x_{F}$. The effect does not show appreciable dependence on $p_{T}$.

*e-mail: jdd@fisica.ist.utl.pt

$\dagger$ †-mail: fduraes@axpfep1.if.usp.br

$\ddagger$ (On leave from Instituto de Física, Universidade de São Paulo - SP, Brazil) 
In QCD, charmed quarks are generated by parton-antiparton fusion and in this process the $c$ quarks are produced with relatively small rapidities such that their fragmentation or coalescence probability is not likely to reproduce the observed asymmetry [2]. Some models, containing recombination mechanism [3, 4, 5, 6], fast $c$-quark strings [7] and intrinsic charm [8, 9] can be adjusted to reproduce data.

In the present paper we argue that the asymmetry may be essentially due to the, so far neglected, $d \rightarrow D^{-}(d \bar{c})$ fragmentation which, for large $x_{F}$, gives the required $D^{-}$dominance.

Experimentally, not much is known about the production of $D$ (or $B$ ) mesons from light quarks. In $e^{+} e^{-}$annihilation, most of the heavy mesons come from heavy quarks. Detection of heavy meson in one hemisphere, with absence of heavy meson in the opposite hemisphere, would be an indication of fragmentation from light quark.

Theoretically, and taking as an example $D^{-}$meson production, if one looks at $e^{+} e^{-}$at $Q=2 m_{D}$ threshold there are two possibilities: production from $d$ quark and production from $\bar{c}$ antiquark (Fig.1). At the $m_{D}$ threshold the two fragmentation functions, $D_{D^{-} / d}\left(z, m_{D}\right)$ and $D_{D^{-} / \bar{c}}\left(z, m_{D}\right)$ are of the form [10, 11],

$$
\begin{aligned}
& D_{D^{-} / d}\left(z, m_{D}\right) \sim \delta(1-z), \\
& D_{D^{-} / \bar{c}}\left(z, m_{D}\right) \sim \delta(1-z),
\end{aligned}
$$

with $z=2 P \cdot Q / Q^{2}, P$ and $Q$ being the four-momenta of the $D$ meson and the virtual photon, respectively. The normalizations in (2) and (3) are naturally different, a larger factor is expected in the $\bar{c}$ antiquark fragmentation. But, in both cases, the $D^{-}$meson takes all the energy available: $z=1$. At the same threshold energy $m_{D}$ the unfavoured fragmentation functions $D_{D^{-} / c}\left(z, m_{D}\right)$ and $D_{D^{-} / \bar{d}}\left(z, m_{D}\right)$ are identically zero.

By making use of threshold energy fragmentation functions and applying QCD non-singlet evolution and (possible) spin and ressonance effects [11], one arrives, at $Q>m_{D}$, to the fragmentation functions:

$$
\begin{gathered}
D_{D^{-/ d}}(z, Q)-D_{D^{-/} / \bar{d}}(z, Q) \\
D_{D^{-} / \bar{c}}(z, Q)-D_{D^{-/ c}}(z, Q) .
\end{gathered}
$$

The key idea is that these two non-singlet fragmentation functions, as QCD is flavour independent, remain similar in shape. The functions (4) and (5) are peaked at an intermediate value of $z$ [11], and can be represented, for instance, by Peterson's parameterization [12]. The main result is that the $d \rightarrow D^{-}$fragmentation will produce, like $\bar{c} \rightarrow D^{-}$fragmentation, fast mesons. Note that a similar behaviour is also observed in $u \rightarrow K^{+}$and $\bar{s} \rightarrow K^{+}$fragmentation functions 13 .

One should notice that in (4), because of charge conjugation, we have the difference between leading and non-leading $D$ mesons, with $\pi^{-}$beams, as it appears in the numerator of the asymmetry (1):

$$
D_{D^{-} / d}(z, Q)-D_{D^{+} / d}(z, Q) .
$$

At large $x_{F}$ the $D$ mesons must come from a fast quark and $x_{F} \simeq z$. This means that, at least for large $x_{F},(6)$ must behave similarly to the Peterson's formula. Within large errors, this is consistent with data, as we shall see later. 
In order to construct the full fragmentation functions, as the perturbative QCD is not simple and is too ambiguous, concerning final states, we shall make use of an old non perturbative model of Krzywicki and Peterson [14], further developed in [15]. The mesons in the quark fragmentation are generated via an integral equation and are produced ordered in rapidity (see Fig.2), such that meson 1 is, on the average, faster than meson 2, etc. If $D_{1}(z)$ is the fragmentation function for the first rank meson, $D_{2}(z)$ the fragmentation function for the second rank meson, etc, we have [15]

$$
D_{2}(z)=\int_{0}^{1-z} D_{1}\left(z^{\prime}\right) D_{1}\left(\frac{z}{1-z^{\prime}}\right) \frac{d z^{\prime}}{1-z^{\prime}}
$$

and, in general,

$$
D_{k}(z)=\int_{0}^{1-z} D_{1}\left(z^{\prime}\right) D_{k-1}\left(\frac{z}{1-z^{\prime}}\right) \frac{d z^{\prime}}{1-z^{\prime}}
$$

The functions $D_{k}(z)$ are normalized to 1 . The full fragmentation function is

$$
D(z)=\sum_{k} D_{k}(z)
$$

As only the leading meson can be of rank 1 , the function $D_{1}(z)$ is nothing but the difference between the leading and non-leading fragmentation functions (see (6)).

It is easily seen that in the limit $z \rightarrow 1$ from (7) and (8) we obtain:

$$
D_{2}(z) \underset{z \rightarrow 1}{\sim} D_{1}(0) D_{1}(z)(1-z)
$$

and

$$
D_{k}(z) \underset{z \rightarrow 1}{\sim} D_{1}(0)^{k-1} D_{1}(z)(1-z)^{k-1} .
$$

In this limit, keeping only the most important terms, $D_{1}$ and $D_{2}$, and identifying $z \simeq x_{F}$ we obtain for the asymmetry (1):

$$
A\left(x_{F}\right)_{x_{F} \rightarrow 1}=\frac{D_{1}\left(x_{F}\right)+D_{2}\left(x_{F}\right)-D_{2}\left(x_{F}\right)}{D_{1}\left(x_{F}\right)+D_{2}\left(x_{F}\right)+D_{2}\left(x_{F}\right)} \simeq 1-2 D_{1}(0)\left(1-x_{F}\right) .
$$

Two remarks can be made regarding Eq. (12). The first one is that the asymmetry increases and approaches 1 as $x_{F} \rightarrow 1$. The second one is that the approach of the $x_{F} \rightarrow 1$ limit is controlled by the behaviour of $D_{1}(z)$ at $z \rightarrow 0$. In order to see the importance of this point let us assume that in the $z \simeq 0$ region the function $D_{1}$ behaves as:

$$
D_{1}(z) \underset{z \rightarrow 0}{\rightarrow} z^{\alpha}
$$

with $\alpha>-1$. The function $D_{2}(z)$, in the $z \rightarrow 1$ limit, will behave as

$$
D_{2}(z) \underset{z \rightarrow 1}{\rightarrow} D_{1}(z)(1-z)^{\alpha+1}
$$

and the asymmetry

$$
A\left(x_{F}\right) \underset{x_{F} \rightarrow 1}{\simeq} 1-c\left(1-x_{F}\right)^{\alpha+1}
$$


$c$ being some normalization constant. By computing the second derivative $d^{2} A / d x_{F}^{2}$ one immediately sees that the $x_{F} \rightarrow 1$ limit is approached with negative curvature if $\alpha>0$, with positive curvature if $-1<\alpha<0$, and in a straight line manner if $\alpha=0$. As we shall see later, data favours a behaviour of $D_{1}(z)$ in the $z \rightarrow 0$ limit with $\alpha \gtrsim 0$, as expected from QCD non-singlet evolution [11] and from Peterson's formula [12].

In order to be somewhat more precise we shall look more carefully to $D^{-}$and $D^{+}$production from $\pi^{-}$beam, keeping the relevant functions, in the $x_{F} \rightarrow 1$ limit, $D_{1}$ and $D_{2}$. We have the contributions of Fig.3. While contributions a) and b) involve the function $D_{1}$ with a charm quark, the contributions c) and d) involve the function $\tilde{D}_{1}$ without charm quark $(d \rightarrow d \bar{u}$ or $\bar{u} \rightarrow \bar{u} d)$. The signals - and + indicate $D^{-}$and $D^{+}$meson production, respectively, but $D_{1}^{+}=D_{1}^{-}$, etc. The factors $1 / 2$ account for isospin (strange quarks are neglected in comparison with $u$ and $d$ quarks). The function $\tilde{D}_{2}$ is written (see Fig.3),

$$
\tilde{D}_{2}(z)=\int_{0}^{1-z} \tilde{D}_{1}\left(z^{\prime}\right) D_{1}\left(\frac{z}{1-z^{\prime}}\right) \frac{d z^{\prime}}{1-z^{\prime}} .
$$

We can finally write the fragmentation functions $D^{-}(z)$ and $D^{+}(z)$, keeping only $D_{1}, D_{2}$ and $\tilde{D}_{2}$ contributions, and identifying again $x_{F} \simeq z$, as

$$
\begin{array}{lr}
D^{-}\left(x_{F}\right)= & D_{1}\left(x_{F}\right)+\frac{1}{2} D_{2}\left(x_{F}\right)+\frac{1}{2} \tilde{D}_{2}\left(x_{F}\right)+\ldots \\
D^{+}\left(x_{F}\right)= & \frac{1}{2} D_{2}\left(x_{F}\right)+\frac{1}{2} \tilde{D}_{2}\left(x_{F}\right)+\ldots
\end{array}
$$

and, for the asymmetry,

$$
A\left(x_{F}\right) \underset{x_{F} \rightarrow 1}{\simeq} \frac{D_{1}\left(x_{F}\right)}{D_{1}\left(x_{F}\right)+D_{2}\left(x_{F}\right)+\tilde{D}_{2}\left(x_{F}\right)+\ldots}
$$

In an attempt to compare (19) with experiment we have used for $D_{1}(z)$ and $\tilde{D}_{1}(z)$ Peterson's parameterization, with the values $\left.\varepsilon=0.06(<z\rangle_{d \bar{c}} \simeq 0.78\right)$ for $D_{1}$ and $\varepsilon \simeq 3.3(<z\rangle_{\bar{u} d} \simeq$ $0.35)$ for $\tilde{D}_{1}$, and $(7)$ and (16) to compute $D_{2}$ and $\tilde{D}_{2}$. Note that the use of Peterson's formula is theoretically not justified for $d \rightarrow d \bar{u}$ or $\bar{u} \rightarrow \bar{u} d$ fragmentation, the reason why we used it is because it reasonably fits data and parametrizations of $\tilde{D}_{1} \equiv 2\left(D_{\pi^{+} / u}-D_{\pi^{-} / u}\right)$ [11, 13, 15].

In Fig.4 we compare directly our formula (19) with data on the $\pi^{-} \rightarrow D^{ \pm}$asymmetry. The agreement is reasonable, better than it should. The limit $A=1$ is approached with negative curvature due to the fact that $D_{1}$ and $\tilde{D}_{1}$, experimentally and in agreement with Peterson's formula, smoothly vanish as $z \rightarrow 0$ (see (13)). Including higher rank $D$ meson production, i.e., $D_{3}, \tilde{D}_{3}, D_{4}, \tilde{D}_{4}$, etc., fragmentation functions, (19) goes to zero faster than in the figure, as $x_{F}$ moves to zero.

It is clear that the comparison of Eq. (19) with data in Fig.4, except in the $x_{F} \rightarrow 1$ region, is far from being justified. In general, one requires the convolution of parton structure functions $f(x)$ with fragmentation functions $D(z)$, with $x_{F}=z x$, at least for the valence $\bar{u}$ and $d$ quarks. In doing so, one realizes that leading particles, $D^{-}$, can be produced even at small $x_{F}$ (from small $x$ quarks) and, in a pure fragmentation approach, as considered here, the asymmetry is not expected to approach zero as $x_{F} \rightarrow 0$.

We believe that in our comparison with data in Fig.4 we are making two, somehow compensating, mistakes. Inclusion of $D_{3}, \tilde{D}_{3}, D_{4}, \tilde{D}_{4}$, etc., contributions would have decreased the 
asymmetry for small $x_{F}$. Inclusion of simultaneous fragmentation of $\bar{u}$ and $d$ would have increased the asymmetry for small $x_{F}$. As we mentioned above our results are better than they should have been, but we think that we understand why is it so.

There is a general result, from our approach which is independent of convolution calculations involving structure functions. If one selects the sample of events where $D^{-}$or/and $D^{+}$are produced, then

$$
<N^{-}>-<N^{+}>=1 / 2
$$

where $\left\langle N^{\mp}>\right.$ is the $D^{\mp}$ average multiplicity in the $D^{-}, D^{+}$sample. If in (20) one takes normal average multiplicities (over all the events), the right hand side of (20) becomes $1 / 2 \sigma^{D} / \sigma_{i n}$., where $\sigma^{D}$ is the cross-section for $D^{ \pm}$production and $\sigma_{i n}$. the inelastic cross-section. In principle, it is not difficult to test experimentally Eq. (20).

In Fig.5 we show our prediction for the $B^{-}, B^{+}$asymmetry. As the $b$ quark fragmentation $D_{1}$ is, in this case, closer to a $\delta$-function $\left(\varepsilon=0.018,<z>_{u \bar{b}} \simeq 0.87\right.$, in Peterson's formula) the asymmetry becomes important closer to $x_{F} \rightarrow 1$.

Concerning $D^{0}, \bar{D}^{0}$ production from $\pi^{-}$beams an asymmetry essentially identical to the $D^{-}, D^{+}$asymmetry is expected. In the case of $D_{s}^{-}, D_{s}^{+}$production, naturally no asymmetry is expected [16].

Our treatment of light quark fragmentation functions into heavy mesons can be easily included in multi-interacting parton models, as the Dual Parton Model [17].

\section{Acknowledgments}

J.D.D. thanks the hospitality at the Instituto de Física, Universidade de S. Paulo, and usefull discussions with Fernando Navarra. F.D. thanks the hospitality at the Departamento de Física and CENTRA, Instituto Superior Técnico, where most of this work was done, and the help from everybody there. F.D. acknowledgs a research grant from CAPES, Brasil. This work was supported by the contract PRAXIS/PCEX/P/FIS/124/96. 


\section{References}

[1] S. Frixione, M. L. Mangano, P. Nason and G. Ridolfi, "Heavy-Quark Production", CERNTH/97-16 (hep-ph/9702287); Nucl. Phys. B431 (1994) 453.

[2] R. Vogt and S. J. Brodsky, Nucl. Phys. B438 (1995) 261.

[3] R.C. Hwa, Phys. Rev. D51 (1995) 85.

[4] F. O. Durães, F. S. Navarra, C. A. A. Nunes and G. Wilk, Phys. Rev. D53 (1996) 6136.

[5] E. Cuantle, G. Herrera and J. Magnin, " $D^{ \pm}$and $D^{0}\left(\bar{D}^{0}\right)$ Production Asymmetries in $\pi p$ Collisions", hep-ph/9711354.

[6] A. K. Likhoded and S. R. Slabopitsky, "On Mechanism of Charmed c-Quarks Fragmentation in Hadronic Collisions", hep-ph/9710476.

[7] T. Sjöstrand, Comp. Phys. Commun. 39 (1986) 344; T. Sjöstrand and W. Bengtsson, Comp. Phys. Commun. 43 (1987) 367.

[8] S. J. Brodsky, P. Hoyer, C. Peterson and N. Sakai, Phys. Lett. B93 (1980) 451; S. J. Brodsky, C. Peterson and N. Sakai, Phys. Rev. D23 (1981) 2745.

[9] O. I. Piskovmova, Nucl. Phys. Proc. (Suppl.) 50 (1996) 179.

[10] H. Georgi and H. D. Politzer, Nucl. Phys. B136 (1978) 445.

[11] J. Dias de Deus and N. Sakai, Phys. Lett. 86B (1979) 321.

[12] C. Peterson, D. Schlalter, I. Schmitt and P. Zerwas, Phys. Rev. D27 (1983) 105.

[13] R. D. Fields and R. P. Feynman, Phys. Rev. D15 (1977) 2590.

[14] A. Krzywicki and B. Petersson, Phys. Rev. D6 (1972) 924.

[15] J. Dias de Deus, Nucl. Phys. B138 (1978) 465.

[16] E.M. Aitala et al., (Fermilab E791 Collaboration), Phys. Lett. B411 (1997) 230.

[17] A. Capella, U. P. Sukhatme, C. I. Tan and J. Tran Thanh Van, Phys. Rep. 236 (1994) 225.

[18] G. A. Alves et al., (E769 Coll.), Phys. Rev. Lett. 77 (1996) 2392.

[19] E. M. Aitala et al., (E791 Coll.), Phys. Lett. B371 (1996) 157.

[20] M. Adamovich et al., (WA82 Coll.), Phys. Lett. B305 (1993) 812.

[21] M. Adamovich et al., (WA92 Coll.), Nucl. Phys. B495 (1997) 3. 


\section{Figure Captions}

Fig. 1 The fragmentation functions, a), $d \rightarrow D^{-}$and, b), $\bar{c} \rightarrow D^{-}$at the $Q=m_{D}$ threshold. The two functions have the same shape in $z$, even after QCD evolution.

Fig. 2 Model to generate fragmentation functions with ordered mesons, rank 1, 2, 3, etc. See Eqs. (7) and (8).

Fig. 3 Fast, rank 1 and rank 2, contributions to the $D^{\mp}$ fragmentation functions. Only diagram a) contributes to the asymmetry.

Fig. 4 Comparison of Eq. (19) for the asymmetry $A\left(x_{F}\right)$ with experimental data [18, 19, 20, 21].

Fig. $5 D^{\mp}$ and $B^{\mp}$ asymmetry as a function of $x_{F}$. 


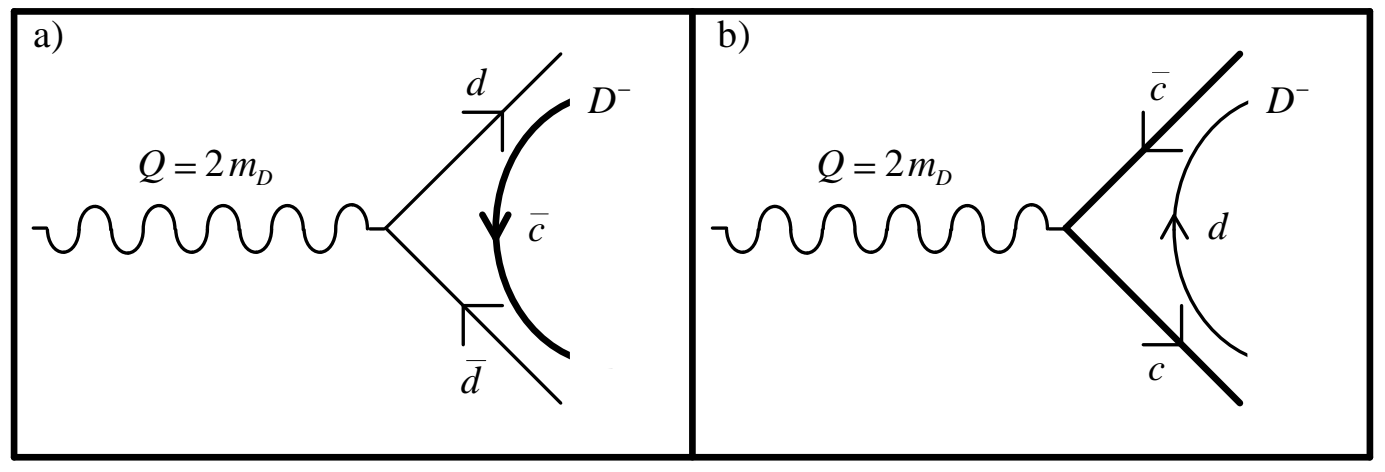

Figure 1 


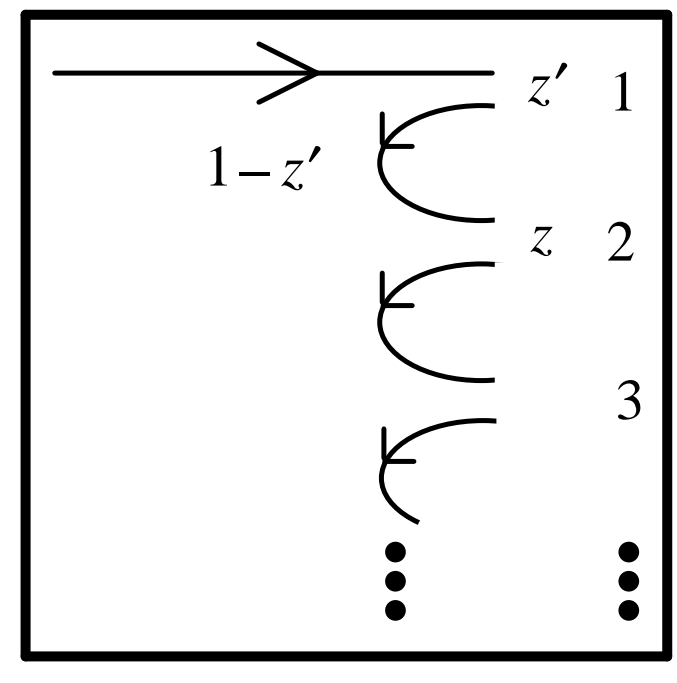

Figure 2 


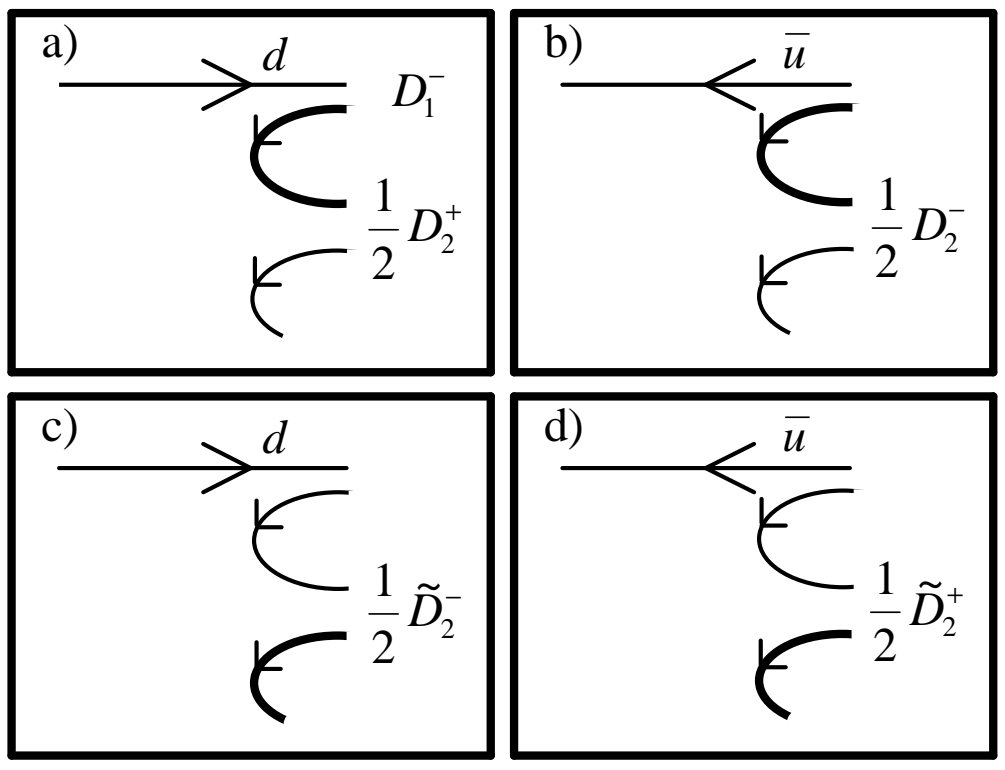

Figure 3 


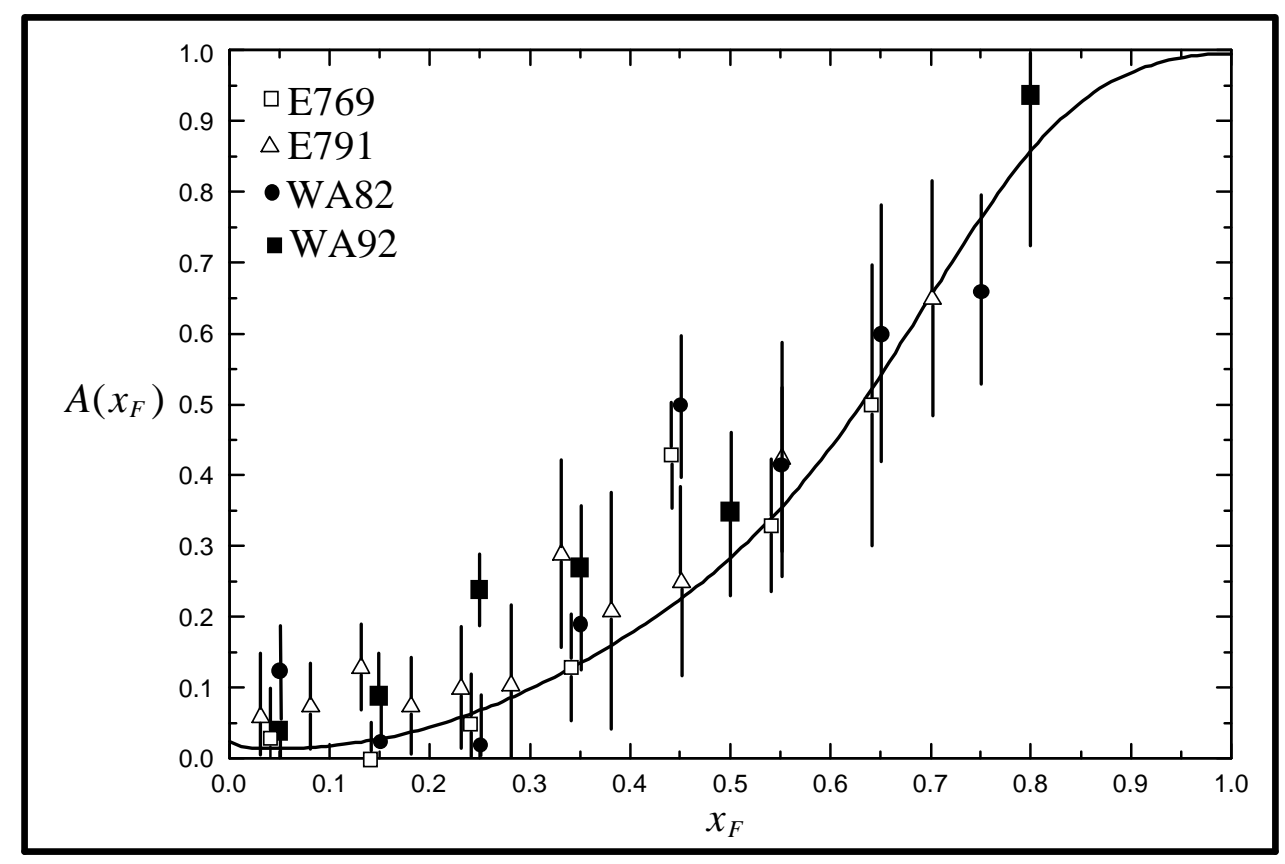

Figure 4 


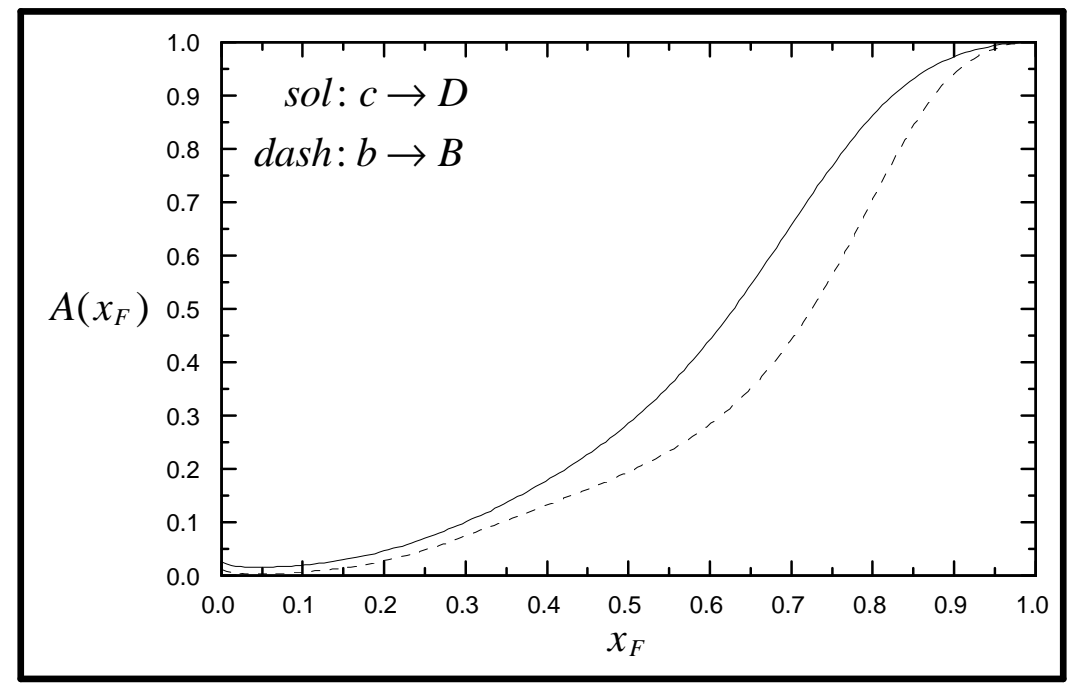

Figure 5 